\title{
Wakefield generation by a relativistic ring beam in a coaxial two-channel dielectric loaded structure
}

\author{
Wanming Liu and Wei Gai \\ High Energy Physics Division, Argonne National Laboratory, Argonne, Illinois 60439, USA
}

(Received 13 March 2009; published 12 May 2009)

\begin{abstract}
In this paper, we give a complete analytical solution for wakefields generated by an azimuthally symmetric ring beam propagating in a coaxial two-channel dielectric structure. This wakefield can be used to accelerate a witness beam in the central channel. The ratio of the peak accelerating field in the center channel to the decelerating field in the ring channel (defined as transformer ratio $R$ ) is also derived. We find that, by appropriate choice of parameters, $R$ can be much greater than 2 , the limiting value for collinear wakefield accelerators.
\end{abstract}

DOI: 10.1103/PhysRevSTAB.12.051301

PACS numbers: 41.60.-m, 41.75.Lx, 94.20.wj

\section{INTRODUCTION}

In a wakefield accelerator, the fields generated by a leading, high-charge drive bunch (either a single drive bunch or a train of drive bunches) are used to accelerate a trailing, low-charge witness bunch. An important parameter that influences the performance of a wakefield accelerator is the transformer ratio $R \equiv$ (maximum energy gain of the witness bunch)/(maximum energy loss of the drive bunch) [1]. Therefore, to accelerate the witness beam to high energy it is desirable to make $R$ as large as possible. There are two major classes of wakefield accelerator geometries, collinear and two beam. For a collinear wakefield accelerator, $R$ is less than 2 under very general conditions: linear media; a relativistic, longitudinally symmetric drive bunch; and identical paths through the system of both drive and witness beams $[2,3]$. Some of the methods that can be employed to obtain $R>2$ include: a triangular longitudinal drive bunch profile [4]; a train of Gaussian drive bunches of progressively increasing charge (ramped bunch train) [5-7]; use of a proton drive beam so that the particles can change positions within the bunch during deceleration [8]; and nonlinear plasma dynamics [9]. Another way to achieve $R>2$ is by designing separate drive and witness beam lines with different shunt impedances $[10,11]$. One possible approach (Fig. 1) is to use a multichannel structure with a ring drive beam propagating through a coaxial outer vacuum channel and a witness beam through a central channel. This technique has been proposed by Weiland for metallic structures [12] and by Hirshfield for dielectric structures [13].

In this paper, we present both analytic and numerical simulation results for the wakefield excited by a ring of charge in a two-channel circular dielectric loaded accelerator (DLA) structure. We begin by solving for the Green's function of the TM modes of the structure and then analyze the first two monopole modes of the wakefield in detail. Numerical simulations using MAFIA [14] and CST MICROWAVE STUDIO [15] are used to verify the analytical results.

\section{THE GREEN'S FUNCTION SOLUTION OF A RING OF CHARGE}

The coaxial, two-channel DLA structure of Fig. 1 has an inner vacuum region $(r<a)$ for the witness beam and an outer vacuum region $(r 1<r<r 2)$ for the drive beam. The drive beam used to excite the DLA is azimuthally $(\varphi)$ symmetric; an important requirement to avoid exciting dipole modes which can cause beam instabilities. In this paper, we will concentrate on the monopole wakefield excitation due to a uniform ring of electrons.

For a uniform, annular electron beam moving at speed $v$, the corresponding wave equations for the longitudinal field components can be obtained from

$$
\begin{aligned}
\nabla^{2} E_{z}-\mu_{0} \varepsilon_{0} \frac{\partial^{2} E_{z}}{\partial t^{2}}= & \frac{\partial}{\partial z}\left[\frac{q \delta\left(r-r_{0}\right) \delta(z-v t)}{\varepsilon_{0} r}\right] \\
& +\frac{\partial}{\partial t}\left[\mu_{0} q v \frac{\delta\left(r-r_{0}\right)}{r} \delta(z-v t)\right] \\
\nabla^{2} B_{z}-\mu_{0} \varepsilon_{0} \frac{\partial^{2} B_{z}}{\partial t^{2}}= & 0 .
\end{aligned}
$$

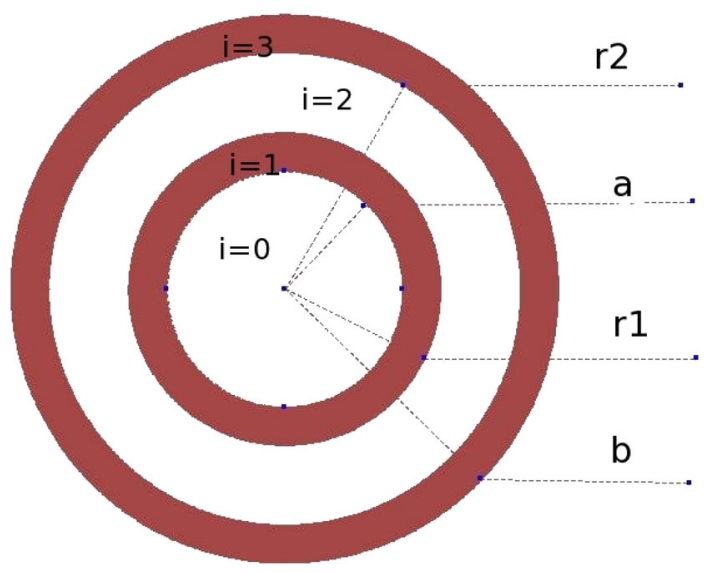

FIG. 1. (Color) Cross section of the coaxial two-channel DLA structure investigated in this paper. 
Since we are only considering the monopole field excited by the drive beam, which is a TM wave with $B_{z}=0$, we only need to solve for $E_{z}$. We can now rewrite Eq. (1) as

$$
\begin{aligned}
& \left(\nabla_{r}^{2}+\frac{\partial^{2}}{\partial z^{2}}\right) E_{z}-\mu_{0} \varepsilon_{0} \frac{\partial^{2} E_{z}}{\partial t^{2}} \\
& =\frac{q \delta\left(r-r_{0}\right) \delta(z-v t)}{\varepsilon_{0} r}\left(1-\mu_{0} \varepsilon_{0} v^{2}\right) \\
& =\frac{q \delta\left(r-r_{0}\right) \delta(z-v t)}{\varepsilon_{0} r \gamma^{2}} .
\end{aligned}
$$

By taking the Fourier transform of Eq. (2) with respect to $z-v t$, we obtain

$$
\begin{aligned}
& \nabla_{r}^{2} \tilde{E}_{z}-\frac{\omega^{2}}{v^{2}}\left(1-v^{2} \mu_{0} \varepsilon_{0}\right) \tilde{E}_{z} \\
& =\frac{q \delta\left(r-r_{0}\right)}{\varepsilon_{0} r \gamma^{2}} \int_{-\infty}^{\infty} \delta^{\prime}(z-v t) e^{j(\omega / v)(z-v t)} d(z-v t) .
\end{aligned}
$$

After applying integration by parts to the integral on the right-hand side, we then have

$$
\nabla_{r}^{2} \tilde{E}_{z}-\frac{\omega^{2}}{v^{2}}\left(1-v^{2} \mu_{0} \varepsilon_{0}\right) \tilde{E}_{z}=\frac{-j \omega q \delta\left(r-r_{0}\right)}{\varepsilon_{0} r v \gamma^{2}},
$$

where $\nabla_{r}^{2}=\frac{\partial^{2}}{\partial r^{2}}+\frac{1}{r} \frac{\partial}{\partial r}$. We next solve for the general and particular solutions of Eq. (3b).

The particular solution of Eq. (3b) can be found by taking the Hankel transform of both sides of Eq. (3b) and denoting the Hankel transform of $\tilde{E}_{z}$ as $\tilde{E}_{z}^{H}$. We then have

$$
\tilde{E}_{z}^{H}=\frac{j \omega q}{\varepsilon_{0} v} \frac{J_{0}\left(k r_{0}\right)}{\left[k^{2} \gamma^{2}+\frac{\omega^{2}}{v^{2}}\right]} .
$$

Next, by taking the inverse Hankel transform of Eq. (4), we arrive at the particular solution of Eq. (3b) as

$$
\tilde{E}_{z}^{\text {part }}(r)=\int \tilde{E}_{z}^{H} J_{0}(k r) k d k=\frac{j \omega q}{\varepsilon_{0} v} \int \frac{J_{0}\left(k r_{0}\right) J_{0}(k r)}{k^{2} \gamma^{2}+\frac{\omega^{2}}{v^{2}}} k d k .
$$

Equation (5) can be integrated to give the particular solution

$$
\tilde{E}_{z}^{\text {part }}(r)=\frac{j \omega q}{\varepsilon_{0} v \gamma^{2}} \begin{cases}I_{0}\left(\frac{\omega}{\gamma v} r_{0}\right) K_{0}\left(\frac{\omega}{\gamma v} r\right) & r>r_{0} \\ I_{0}\left(\frac{\omega}{\gamma v} r\right) K_{0}\left(\frac{\omega}{\gamma v} r_{0}\right) & r<r_{0} .\end{cases}
$$

For the general solution of Eq. (3b) we have

$$
\tilde{E}_{z, i}^{\mathrm{gen}}(r)=A_{i} I_{0}\left(k_{r, i} r\right)+B_{i} K_{0}\left(k_{r, i} r\right),
$$

where $i=0$ and 2 refer to the vacuum channel regions. For the dielectric loaded regions 1 and 3, Eq. (3b) becomes

$$
-\left[k^{2}+\frac{\omega^{2}}{v^{2}}\left(1-\frac{v^{2}}{c^{2}} \mu_{r} \varepsilon_{r}\right)\right] \tilde{E}_{z}^{H}=0 .
$$

If we choose the material properties such that $1-$ $\frac{v^{2}}{c^{2}} \mu_{r} \varepsilon_{r}<0$, then the general solution will be a linear superposition of Bessel functions,

$$
\tilde{E}_{z, i}^{\mathrm{gen}}(r)=A_{i} J_{0}\left(k_{r, i} r\right)+B_{i} Y_{0}\left(k_{r, i} r\right),
$$

where $i=1$ and 3 , and $k_{r, i}=\frac{\omega}{v} \sqrt{\frac{v^{2}}{c^{2}} \mu_{r} \varepsilon_{r}-1}$.

Adding the particular solution to the general solution of Eq. (3b) in the drive channel (region 2), we obtain the complete solution of Eq. (3b) in the drive channel as

$$
\begin{aligned}
\tilde{E}_{z, 2}(r)= & A_{2} I_{0}\left(k_{r, 2} r\right)+B_{2} K_{0}\left(k_{r, 2} r\right) \\
& +\frac{j \omega q}{\varepsilon_{0} v \gamma^{2}} \begin{cases}I_{0}\left(\frac{\omega}{\gamma v} r_{0}\right) K_{0}\left(\frac{\omega}{\gamma v} r\right) & r>r_{0} \\
I_{0}\left(\frac{\omega}{\gamma v} r\right) K_{0}\left(\frac{\omega}{\gamma v} r_{0}\right) & r<r_{0} .\end{cases}
\end{aligned}
$$

Equations (7), (9), and (10) together give the Fourier transform of the Green's function of the ring beam wakefield excitation in coaxial two-channel DLA structures. The next step is to determine the coefficients $A_{i}$ and $B_{i}$ by imposing the boundary conditions.

\section{SOLUTION FOR THE COEFFICIENTS AND DERIVATION OF THE DISPERSION RELATION}

Since $E_{z}$ must be finite at $r=0$, then $B_{0}=0$ from Eq. (7). Using the boundary conditions that $E_{z}$ is continuous at $r=a, r_{1}$, and $r_{2}$ and $E_{z}$ is zero on $r=b$, we have the following set of equations:

$$
\begin{aligned}
A_{0} I_{0}\left(k_{r, 0} a\right) & =A_{1} J_{0}\left(k_{r, 1} a\right)+B_{1} Y_{0}\left(k_{r, 1} a\right) \\
A_{1} J_{0}\left(k_{r, 1} r_{1}\right)+B_{1} Y_{0}\left(k_{r, 1} r_{1}\right) & =A_{2} I_{0}\left(k_{r, 2} r_{1}\right)+B_{2} K_{0}\left(k_{r, 2} r_{1}\right)+\frac{j \omega \omega}{\varepsilon_{0} v \gamma^{2}} I_{0}\left(\frac{\omega}{\gamma v} r_{1}\right) K_{0}\left(\frac{\omega}{\gamma v} r_{0}\right) \\
A_{2} I_{0}\left(k_{r, 2} r_{2}\right)+B_{2} K_{0}\left(k_{r, 2} r_{2}\right)+\frac{j \omega q}{\varepsilon_{0} v \gamma^{2}} I_{0}\left(\frac{\omega}{\gamma v} r_{0}\right) K_{0}\left(\frac{\omega}{\gamma v} r_{2}\right) & =A_{3} J_{0}\left(k_{r, 3} r_{2}\right)+B_{3} Y_{0}\left(k_{r, 3} r_{2}\right) \\
A_{3} J_{0}\left(k_{r, 3} b\right)+B_{3} Y_{0}\left(k_{r, 3} b\right) & =0 .
\end{aligned}
$$

Using the boundary conditions of $B_{\phi}$ at $r=a, r_{1}$, and $r_{2}$, we have the following equations: 


$$
\begin{aligned}
& \frac{-j \omega \varepsilon_{0}}{k_{r, 0}} A_{0} I_{1}\left(k_{r, 0} a\right)= \frac{-j \omega \varepsilon_{1}}{k_{r, 1}}\left[A_{1} J_{1}\left(k_{r, 1} a\right)+B_{1} Y_{1}\left(k_{r, 1} a\right)\right] \\
& \frac{-j \omega \varepsilon_{1}}{k_{r, 1}}\left[A_{1} J_{1}\left(k_{r, 1} r_{1}\right)+B_{1} Y_{1}\left(k_{r, 1} r_{1}\right)\right]=-\frac{j \omega \varepsilon_{2}}{k_{r, 2}}\left[A_{2} I_{1}\left(k_{r, 2} r_{1}\right)+B_{2} K_{1}\left(k_{r, 2} r_{1}\right)\right.\left.+\frac{j \omega q}{\varepsilon_{0} v \gamma^{2}} I_{1}\left(k_{r, 2} r_{1}\right) K_{0}\left(k_{r, 2} r_{0}\right)\right] \\
& \frac{-j \omega \varepsilon_{2}}{k_{r, 2}}\left[A_{2} I_{1}\left(k_{r, 2} r_{2}\right)+B_{2} K_{1}\left(k_{r, 2} r_{2}\right)+\frac{j \omega q}{\varepsilon_{0} v \gamma^{2}} I_{0}\left(k_{r, 2} r_{0}\right) K_{1}\left(k_{r, 2} r_{2}\right)\right]=-\frac{j \omega \varepsilon_{3}}{k_{r, 3}}\left[A_{3} J_{1}\left(k_{r, 2} r_{2}\right)+B_{3} Y_{1}\left(k_{r, 3} r_{2}\right)\right] .
\end{aligned}
$$

The solution will be determined by solving Eqs. (11a) and (11b) together. The combination of Eqs. (11a) and (11b) can be reduced to the following two equations with two unknowns, $A_{2}$ and $B_{2}$ :

$$
F_{11} A_{2}+F_{12} B_{2}=C_{1} \quad F_{21} A_{2}+F_{22} B_{2}=C_{2},
$$

where

$$
\begin{aligned}
& F_{11}=I_{0}\left(k_{r, 2} r_{1}\right) \frac{j \omega \varepsilon_{1}}{k_{r, 1}}\left[F(a) J_{1}\left(k_{r, 1} r_{1}\right)+G(a) Y_{1}\left(k_{r, 1} r_{1}\right)\right]-\frac{j \omega \varepsilon_{2}}{k_{r, 2}} I_{1}\left(k_{r, 2} r_{1}\right)\left[F(a) J_{0}\left(k_{r, 1} r_{1}\right)+G(a) Y_{0}\left(k_{r, 1} r_{1}\right)\right] \\
& F_{12}=K_{0}\left(k_{r, 2} r_{1}\right) \frac{j \omega \varepsilon_{1}}{k_{r, 1}}\left[F(a) J_{1}\left(k_{r, 1} r_{1}\right)+G(a) Y_{1}\left(k_{r, 1} r_{1}\right)\right]-\frac{j \omega \varepsilon_{2}}{k_{r, 2}} K_{1}\left(k_{r, 2} r_{1}\right)\left[F(a) J_{0}\left(k_{r, 1} r_{1}\right)+G(a) Y_{0}\left(k_{r, 1} r_{1}\right)\right] \\
& C_{1}=\frac{-j \omega q}{\varepsilon_{0} v \gamma^{2}} I_{0}\left(k_{r, 2} r_{1}\right) K_{0}\left(k_{r, 2} r_{0}\right) \frac{j \omega \varepsilon_{1}}{k_{r, 1}}\left[F(a) J_{1}\left(k_{r, 1} r_{1}\right)+G(a) Y_{1}\left(k_{r, 1} r_{1}\right)\right] \\
& -\frac{\omega^{2} q}{k_{r, 2} v \gamma^{2}} I_{1}\left(k_{r, 2} r_{1}\right) K_{0}\left(k_{r, 2} r_{0}\right)\left[F(a) J_{0}\left(k_{r, 1} r_{1}\right)+G(a) Y_{0}\left(k_{r, 1} r_{1}\right)\right] \\
& F_{21}=I_{0}\left(k_{r, 2} r_{2}\right) \frac{j \omega \varepsilon_{3}}{k_{r, 3}}\left[J_{1}\left(k_{r, 3} r_{2}\right)-\frac{J_{0}\left(k_{r, 3} b\right)}{Y_{0}\left(k_{r, 3} b\right)} Y_{1}\left(k_{r, 3} r_{2}\right)\right]-\frac{j \omega \varepsilon_{2}}{k_{r, 2}} I_{1}\left(k_{r, 2} r_{2}\right)\left[J_{0}\left(k_{r, 3} r_{2}\right)-\frac{J_{0}\left(k_{r, 3} b\right)}{Y_{0}\left(k_{r, 3} b\right)} Y_{0}\left(k_{r, 3} r_{2}\right)\right] \\
& F_{22}=K_{0}\left(k_{r, 2} r_{2}\right) \frac{j \omega \varepsilon_{3}}{k_{r, 3}}\left[J_{1}\left(k_{r, 3} r_{2}\right)-\frac{J_{0}\left(k_{r, 3} b\right)}{Y_{0}\left(k_{r, 3} b\right)} Y_{1}\left(k_{r, 3} r_{2}\right)\right]-\frac{j \omega \varepsilon_{2}}{k_{r, 2}} K_{1}\left(k_{r, 2} r_{2}\right)\left[J_{0}\left(k_{r, 3} r_{2}\right)-\frac{J_{0}\left(k_{r, 3} b\right)}{Y_{0}\left(k_{r, 3} b\right)} Y_{0}\left(k_{r, 3} r_{2}\right)\right] \\
& C_{2}=\frac{-j \omega q}{\varepsilon_{0} v \gamma^{2}} I_{0}\left(k_{r, 2} r_{0}\right) K_{0}\left(k_{r, 2} r_{2}\right) \frac{j \omega \varepsilon_{3}}{k_{r, 3}}\left[J_{1}\left(k_{r, 3} r_{2}\right)-\frac{J_{0}\left(k_{r, 3} b\right)}{Y_{0}\left(k_{r, 3} b\right)} Y_{1}\left(k_{r, 3} r_{2}\right)\right] \\
& -\frac{\omega^{2} q}{k_{r, 2} v \gamma^{2}} I_{0}\left(k_{r, 2} r_{0}\right) K_{1}\left(k_{r, 2} r_{2}\right)\left[J_{0}\left(k_{r, 3} r_{2}\right)-\frac{J_{0}\left(k_{r, 3} b\right)}{Y_{0}\left(k_{r, 3} b\right)} Y_{0}\left(k_{r, 3} r_{2}\right)\right] \\
& F(a)=\frac{I_{0}\left(k_{r, 0} a\right) Y_{1}\left(k_{r, 1} a\right)-\frac{\varepsilon_{0} k_{r, 1}}{k_{r, 0} \varepsilon_{1}} I_{1}\left(k_{r, 0} a\right) Y_{0}\left(k_{r, 1} a\right)}{J_{0}\left(k_{r, 1} a\right) Y_{1}\left(k_{r, 1} a\right)-J_{1}\left(k_{r, 1} a\right) Y_{0}\left(k_{r, 1} a\right)} \quad G(a)=\frac{I_{0}\left(k_{r, 0} a\right) J_{1}\left(k_{r, 1} a\right)-\frac{\varepsilon_{0} k_{r, 1}}{k_{r, 0} \varepsilon_{1}} I_{1}\left(k_{r, 0} a\right) J_{0}\left(k_{r, 1} a\right)}{Y_{0}\left(k_{r, 1} a\right) J_{1}\left(k_{r, 1} a\right)-Y_{1}\left(k_{r, 1} a\right) J_{0}\left(k_{r, 1} a\right)} .
\end{aligned}
$$

Finally, we can solve for the two unknowns $A_{2}$ and $B_{2}$,

$$
A_{2}=\frac{C_{1} F_{22}-C_{2} F_{12}}{F_{11} F_{22}-F_{21} F_{12}} \quad B_{2}=\frac{C_{1} F_{21}-C_{2} F_{11}}{F_{11} F_{22}-F_{21} F_{12}} .
$$

\section{SPECIAL CASE $\boldsymbol{v} \rightarrow \boldsymbol{c}$}

If we consider the special case when the speed of beam approaches $c$, then we have $k_{r, 2} \rightarrow 0$. Using small argument approximations of Bessel functions, one can rewrite the above (12b)-(12h) as 


$$
\begin{aligned}
F_{11}= & \frac{j \omega \varepsilon_{1}}{k_{r, 1}}\left[F(a) J_{1}\left(k_{r, 1} r_{1}\right)+G(a) Y_{1}\left(k_{r, 1} r_{1}\right)\right]-\frac{j \omega \varepsilon_{2} r_{1}}{2}\left[F(a) J_{0}\left(k_{r, 1} r_{1}\right)+G(a) Y_{0}\left(k_{r, 1} r_{1}\right)\right] \\
F_{12}= & -\ln \frac{\left(k_{r, 2} r_{1}\right)}{2} \frac{j \omega \varepsilon_{1}}{k_{r, 1}}\left[F(a) J_{1}\left(k_{r, 1} r_{1}\right)+G(a) Y_{1}\left(k_{r, 1} r_{1}\right)\right]+\frac{j \omega \varepsilon_{2}}{k_{r, 2}^{2} r_{1}}\left[F(a) J_{0}\left(k_{r, 1} r_{1}\right)+G(a) Y_{0}\left(k_{r, 1} r_{1}\right)\right] \\
C_{1}= & \frac{-j \omega q}{\varepsilon_{0} v \gamma^{2}}\left(-\ln \frac{k_{r, 2} r_{0}}{2}\right) \frac{j \omega \varepsilon_{1}}{k_{r, 1}}\left[F(a) J_{1}\left(k_{r, 1} r_{1}\right)+G(a) Y_{1}\left(k_{r, 1} r_{1}\right)\right]-\frac{\omega^{2} q r_{1}}{2 v \gamma^{2}}\left(-\ln \frac{k_{r, 2} r_{0}}{2}\right) \\
& \times\left[F(a) J_{0}\left(k_{r, 1} r_{1}\right)+G(a) Y_{0}\left(k_{r, 1} r_{1}\right)\right] \\
F_{21}= & \frac{j \omega \varepsilon_{3}}{k_{r, 3}}\left[J_{1}\left(k_{r, 3} r_{2}\right)-\frac{J_{0}\left(k_{r, 3} b\right)}{Y_{0}\left(k_{r, 3} b\right)} Y_{1}\left(k_{r, 3} r_{2}\right)\right]-\frac{j \omega \varepsilon_{2} r_{2}}{2}\left[J_{0}\left(k_{r, 3} r_{2}\right)-\frac{J_{0}\left(k_{r, 3} b\right)}{Y_{0}\left(k_{r, 3} b\right)} Y_{0}\left(k_{r, 3} r_{2}\right)\right] \\
F_{22}= & -\ln \frac{k_{r, 2} r_{2}}{2} \frac{j \omega \varepsilon_{3}}{k_{r, 3}}\left[J_{1}\left(k_{r, 3} r_{2}\right)-\frac{J_{0}\left(k_{r, 3} b\right)}{Y_{0}\left(k_{r, 3} b\right)} Y_{1}\left(k_{r, 3} r_{2}\right)\right]+\frac{j \omega \varepsilon_{2}}{k_{r, 2}^{2} r_{2}}\left[J_{0}\left(k_{r, 3} r_{2}\right)-\frac{J_{0}\left(k_{r, 3} b\right)}{Y_{0}\left(k_{r, 3} b\right)} Y_{0}\left(k_{r, 3} r_{2}\right)\right] \\
C_{2}= & \frac{-j \omega q}{\varepsilon_{0} v \gamma^{2}}\left(-\ln \frac{k_{r, 2} r_{2}}{2}\right) \frac{j \omega \varepsilon_{3}}{k_{r, 3}}\left[J_{1}\left(k_{r, 3} r_{2}\right)-\frac{J_{0}\left(k_{r, 3} b\right)}{Y_{0}\left(k_{r, 3} b\right)} Y_{1}\left(k_{r, 3} r_{2}\right)\right]-\frac{\omega^{2} q}{k_{r, 2}^{2} r_{2} v \gamma^{2}}\left[J_{0}\left(k_{r, 3} r_{2}\right)-\frac{J_{0}\left(k_{r, 3} b\right)}{Y_{0}\left(k_{r, 3} b\right)} Y_{0}\left(k_{r, 3} r_{2}\right)\right] \\
F(a)= & \frac{Y_{1}\left(k_{r, 1} a\right)-\frac{\varepsilon_{0} k_{r, 1} a}{2 \varepsilon_{1}} Y_{0}\left(k_{r, 1} a\right)}{J_{0}\left(k_{r, 1} a\right) Y_{1}\left(k_{r, 1} a\right)-J_{1}\left(k_{r, 1} a\right) Y_{0}\left(k_{r, 1} a\right)} \quad G(a)=\frac{J_{1}\left(k_{r, 1} a\right)-\frac{\varepsilon_{0} k_{r, 1} a}{2 \varepsilon_{1}} J_{0}\left(k_{r, 1} a\right)}{J_{0}\left(k_{r, 1} a\right) Y_{1}\left(k_{r, 1} a\right)-J_{1}\left(k_{r, 1} a\right) Y_{0}\left(k_{r, 1} a\right)}
\end{aligned}
$$

In the special case that $v$ approaches $c, F_{12}$ and $F_{22}$ approach infinity and we rewrite Eq. (13) as

$$
\begin{aligned}
A_{2} & =\frac{C_{1}-C_{2} F_{12} / F_{22}}{F_{11}-F_{21} F_{12} / F_{22}} \\
B_{2} & =\frac{C_{1} F_{21} / F_{22}-C_{2} F_{11} / F_{22}}{F_{11}-F_{21} F_{12} / F_{22}} .
\end{aligned}
$$

Since $C_{1}, F_{21}, C_{2}$, and $F_{11}$ have bounded values, $B_{2}$ will tend to 0 when $v$ is approaching $c$. The field ratio between the witness channel and the drive channel is then determined as

$$
\frac{A_{0}}{A_{2}}=\frac{1}{F(a) J_{0}\left(k_{r, 1} r_{1}\right)+G(a) Y_{0}\left(k_{r, 1} r_{1}\right)} .
$$

Given the definition of the transformer ratio $R$ as the ratio of the acceleration field experienced by the witness beam to the deceleration field experienced by the drive beam [5], one has

$$
R=2 \frac{A_{0}}{A_{2}}
$$

for the ideal case.

Finally, in order to obtain the time and space dependent Green's function in the drive channel, one needs to take the inverse Fourier transform of Eq. (10) which yields

$$
\begin{aligned}
E_{z}(r, z, t) & =\int_{-\infty}^{\infty} \tilde{E}_{z}(r) e^{-j(\omega / v)(z-v t)} d \omega \\
& =\left.2 \pi i \sum \operatorname{Res}\left[\tilde{E}(r) e^{-j(\omega / v)(z-v t)}\right]\right|_{\omega=\omega_{i}},
\end{aligned}
$$

where $\omega_{i}$ is the $i$ th pole in the complex plane of $\omega$ and is determined by

$$
F_{11}-F_{21} F_{12} / F_{22}=0 \text {. }
$$

Equation (18) also determines the dispersion relations of the modes.

\section{COMPARISON OF NUMERICAL SIMULATIONS AND ANALYTIC RESULTS}

Using the above equations, we solve for the first two monopole modes excited by a ring drive beam inside a twochannel DLA structure with $a=2 \mathrm{~mm}, r 1=4.2 \mathrm{~mm}$, $r 2=13.215 \mathrm{~mm}, b=14 \mathrm{~mm}$, and $\varepsilon_{r}=4.76$. The electromagnetic parameters of the first two modes are summarized in Table I. As shown in the table, the excitation of the $\mathrm{TM}_{02}$ mode is 6 times stronger than the $\mathrm{TM}_{01}$ mode in the drive channel and about 4 times stronger than in the witness channel. Thus, the transformer ratio is dominated by the $\mathrm{TM}_{02}$ mode and is about 4.5.

As shown in Fig. 2, the first two poles are located at 15.561 and $24.95 \mathrm{GHz}$, corresponding to the $\mathrm{TM}_{01}$ and $\mathrm{TM}_{02}$ modes. Since the wakefield excited by the beam has to be synchronized to the beam, the phase velocity has to be matched to the beam velocity which in the case of an electron accelerator is approximately equal to the speed of light. This gives us an easy way to verify the analytic

TABLE I. Electromagnetic parameters of the $\mathrm{TM}_{01}$ and $\mathrm{TM}_{02}$ modes excited by an annular beam.

\begin{tabular}{lll}
\hline \hline & $\mathrm{TM}_{01}$ & $\mathrm{TM}_{02}$ \\
\hline Frequency $(\mathrm{GHz})$ & 15.563 & 24.95 \\
$v_{g}$ & $0.817 \mathrm{c}$ & $0.69 \mathrm{c}$ \\
Transformer ratio & 8.1517 & 4.5761 \\
$A_{2}$ & $7.342 \times 10^{12}$ & $4.5717 \times 10^{13}$ \\
\hline \hline
\end{tabular}




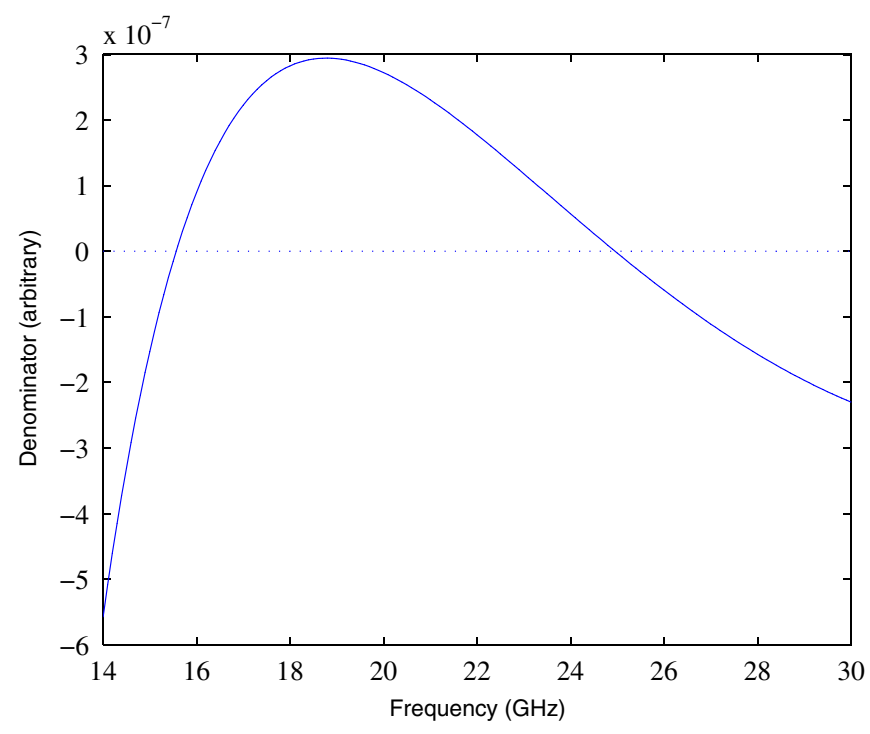

FIG. 2. (Color) The denominator of Eq. (14h) as a function of frequency. Zero crossing at about 15.6 and $25 \mathrm{GHz}$ corresponds to the first two poles of the complex solution.

results with numerical simulations using the commercial software package CST MICROWAVE STUDIO [12]. The dispersion curves calculated with MICROWAVE STUDIO agree well with the analytical results as is shown in Fig. 3 (Fig. 4) for the $\mathrm{TM}_{01}\left(\mathrm{TM}_{02}\right)$ mode.

As a further check, MAFIA [13] was used to calculate the wakefield integral excited by a $2 \mathrm{~mm}$ Gaussian bunch driving a one meter long, coaxial, two-channel DLA (Fig. 5). Using the MAFIA simulation results, we can compare the wavelength, group velocity, and transformer ratio to the analytic results above: (i) wavelength: by measuring the wavelengths in Fig. 5, the frequencies are estimated to be about 15 and $25 \mathrm{GHz}$; (ii) group velocities: using a first order approximation, the relation between group velocity and the slope of envelop is

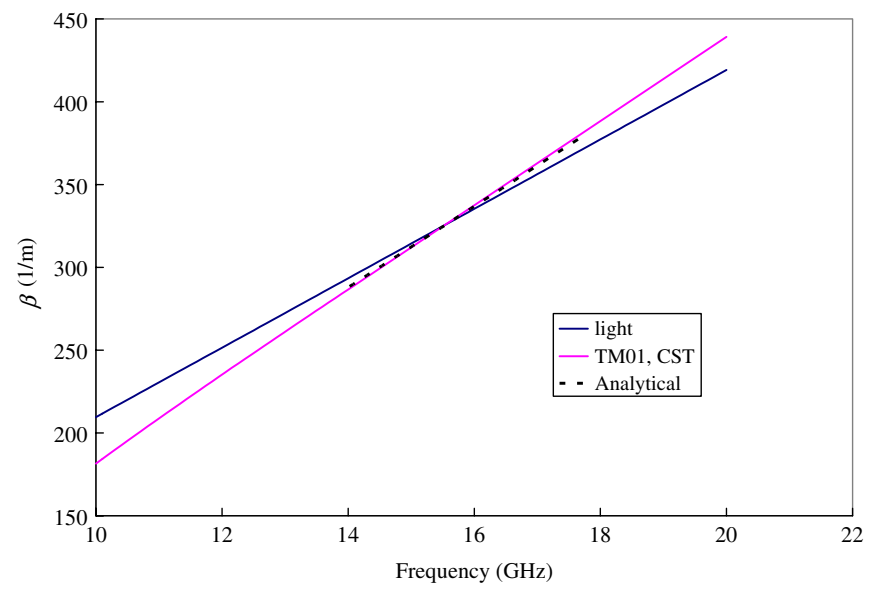

FIG. 3. (Color) Comparison of the analytic and numerical dispersion curves for the $\mathrm{TM}_{01}$ mode. The numerical simulation result is almost identical to the analytic result.

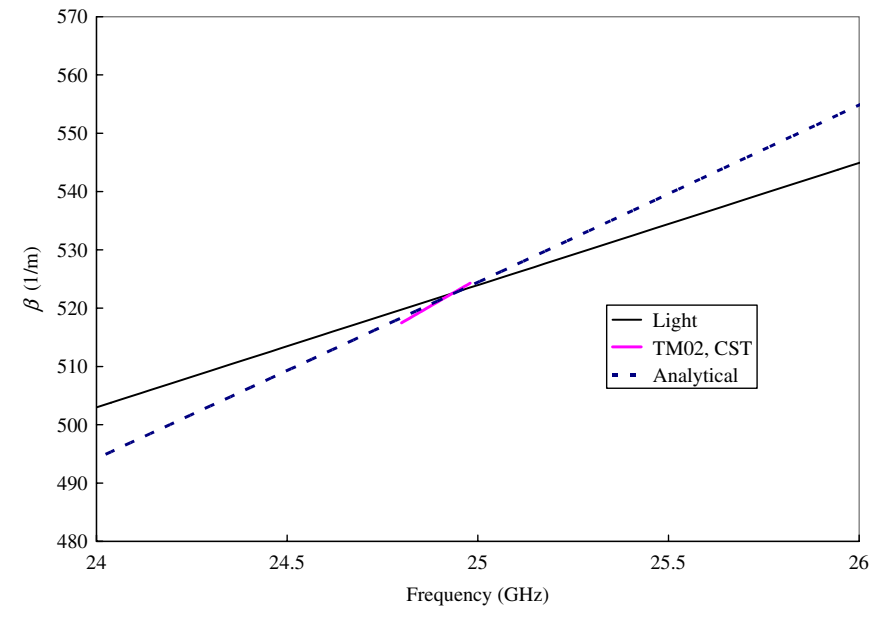

FIG. 4. (Color) Comparison of the analytic and numerical dispersion curves for $\mathrm{TM}_{02}$ mode. The numerical simulation yields a smaller group velocity.

$$
\left(1-v_{g} \cdot d l\right)^{n / 2}=\Delta E,
$$

where $d l$ is a small increment in distance, and $\Delta E$ is the relative change of the field amplitude after $n$ steps. From Fig. 5, the group velocity of the $\mathrm{TM}_{01}$ mode is estimated as $0.82 \mathrm{c}$ and for the $\mathrm{TM}_{01}$ mode as $\sim 0.44 \mathrm{c}$, in good agreement with the analytic predictions. (iii) The transformer

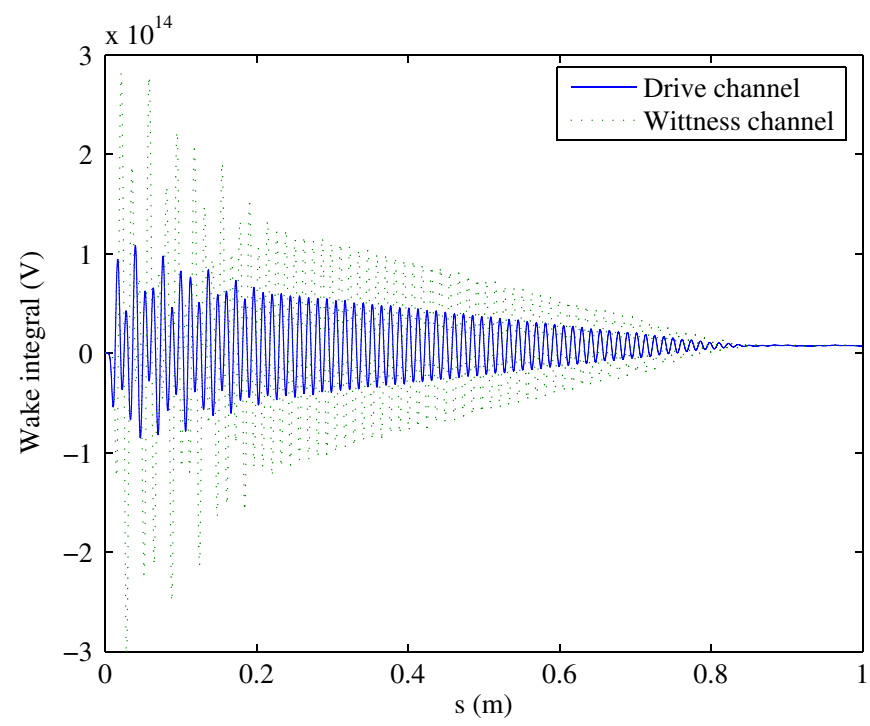

FIG. 5. (Color) MAFIA wakefield simulation results clearly showing two frequency components that correspond to the $\mathrm{TM}_{01}$ and $\mathrm{TM}_{02}$ modes. By measuring the wavelength from this simulation, we estimated the frequencies to be about 15 and $25 \mathrm{GHz}$. From this figure, the low frequency component only shows up in the first quarter and this means the low frequency component has a higher group velocity. The group velocity of the $\mathrm{TM}_{01}$ mode is estimated as $0.82 \mathrm{c}$ and for the $\mathrm{TM}_{01}$ mode as $\sim 0.44 \mathrm{c}$ using Eq. (19). The transformer ratio can also be determined from this plot as 2.6/0.6 4.3 which is in good agreement with the analytical prediction of 4.5 . 
ratio can also be determined from this plot as 2.6/0.6 4.3 which is in good agreement with the analytical prediction of 4.5 .

\section{SUMMARY}

We derived analytical formulas for calculating the wakefield in a coaxial, two-channel DLA structure excited by a ring beam. We checked these formulas against numerical simulations and they are found to be in good agreement with each other. The formulas presented in this paper can be used to design and analyze a coaxial two-channel DLA structure with a transformer ratio greater than 2 .

\section{ACKNOWLEDGMENTS}

We would like to thank Dr. Jay Hirshfield of Yale University for suggesting the coaxial dielectric wakefield experiment and bringing it to our attention. This work is supported by the High Energy Physics Division, DOE under Contract No. DE-AC02-06CH11357.

[1] K. L. Bane, P. Chen, and P. B. Wilson, SLAC Report No. SLAC-PUB-3662, 1985.

[2] P. B. Wilson, in Proceedings of the 13th SLAC Summer Institute On Particle Physics (SLAC Report No. 296), pp. 273-295.

[3] J. G. Power, W. Gai, and P. Schoessow, Phys. Rev. E 60, 6061 (1999).
[4] K. L. Bane, P. Chen, and P. B. Wilson, IEEE Trans. Nucl. Sci. 32, 3524 (1985).

[5] P. Schutt, T. Weiland, and V. M. Tsakanov, in Proceedings of the Nor Amberb Conference, Armenia (1989), Vol. 7, p. 12.

[6] A. G. Ruggiero, P. Schoessow, and J. Simpson, in Proceedings of the Advanced Accelerator Concepts: $3 r d$ Workshop, edited by F.E. Mills (American Institute of Physics, Madison, WI, 1986), p. 247.

[7] C. Jing, A. Kanareykin, J. G. Power, M. Conde, Z. Yusof, P. Schoessow, and W. Gai, Phys. Rev. Lett. 98, 144801 (2007).

[8] J. B. Rosenzweig, P. Schoessow, B. Cole, W. Gai, R. Konecny, J. Norem, and J. Simpson, Phys. Rev. A 39, 1586 (1989).

[9] G. Flesherand and G. Cohn, AIEE Trans. 70, 887 (1955).

[10] G. V. Sotnikov, I. N. Onishchenko, J. L. Hirshfield, and T. C. Marshall, Nucl. Phys. Invest. 49, 148 (2008).

[11] C. Wang and J. L. Hirshfield, Phys. Rev. ST Accel. Beams 9, 031301 (2006).

[12] T. Weiland, KEK Report No. 84-1, 1984.

[13] G. V. Sotnikov, T. C. Marshall, S. V. Shchelkunov, A. Didenko, and J.L. Hirshfield, in Proceedings of the Advanced Accelerator Concepts: 13th Workshop, Santa Cruz, California, 2008 (American Institute of Physics, Melville, New York, 2009), p. 415

[14] http://www.cst.com/Content/Products/MWS/ Overview.aspx.

[15] http://www.cst.com/Content/Products/MAFIA/ Overview.aspx. 